\title{
Immediate synergistic effect of a trunk orthosis with joints providing resistive force and an ankle-foot orthosis on hemiplegic gait
}

This article was published in the following Dove Press journal:

Clinical Interventions in Aging

\author{
Junji Katsuhira' ${ }^{1,2}$ \\ Sumiko Yamamoto ${ }^{3}$ \\ Nodoka Machida ${ }^{4}$ \\ Yuji Ohmura ${ }^{5}$ \\ Masako Fuchi ${ }^{6}$ \\ Mizuho Ohta ${ }^{7}$ \\ Setsuro Ibayashi ${ }^{7}$ \\ Arito Yozu ${ }^{8}$ \\ Ko Matsudaira ${ }^{2}$
}

'Department of Prosthetics and Orthotics and Assistive Technology, Faculty of Medical Technology, Niigata University of Health and Welfare, Niigata, ${ }^{2}$ Department of Medical Research and Management for Musculoskeletal Pain, 22nd Century Medical and Research Center, The University of Tokyo, Tokyo, ${ }^{3}$ Graduate School of International University of Health and Welfare, Tokyo, ${ }^{4}$ International University of Health and Welfare, School of Health Sciences at Narita, Department of Physical Therapy, Chiba, ${ }^{5}$ International University of Health and Welfare, School of Health Sciences at Odawara, Department of Physical Therapy, Kanagawa, ${ }^{6}$ Department of Occupational Therapy, Kyusyu Nutrition Welfare University, Fukuoka, ${ }^{7}$ Seiai Rehabilitation Hospital, Fukuoka, ${ }^{8}$ Center for Medical Sciences, Ibaraki Prefectural University of Health Science, Ibaraki, Japan

Correspondence: Junji Katsuhira Department of Prosthetics and Orthotics and Assistive Technology, Faculty of Medical Technology, Niigata University of Health and Welfare, I398 Shimamichou, Kita-ku, Niigata, Niigata 950-3198, Japan Tel +8I 025257462 I

Fax +8I 025257462 I

Email katsuhira@nuhw.ac.jp
Purpose: The synergistic effects of a trunk orthosis and an ankle-foot orthosis (AFO) in stroke patients with a hemiplegic gait are unclear. We previously developed a trunk orthosis with joints providing resistive force (TORF) to modify malalignment of the trunk and pelvis and confirmed its positive effects in stroke patients during level walking without an AFO. The aim of the present study was to determine if this trunk orthosis and an AFO have synergistic effects during level walking in community-dwelling patients with chronic stroke.

Methods: Twenty-eight community-dwelling stroke patients performed level walking at a selfselected speed with an AFO and again while wearing a TORF (TORF group) or a corset (control group). Spatiotemporal, kinematic, and kinetic data were recorded using a three-dimensional motion analysis system.

Results: When compared with the control group, the TORF group showed significant increases in walking speed, number of steps on the paretic leg per minute, and peak ankle plantar flexion moment during the single stance phase.

Conclusion: The TORF increased the ankle joint plantar flexion moment at the end of the single stance phase during level walking in stroke patients, leading to an increase in their gait speed because of the modified trunk and pelvis alignment.

Keywords: biomechanics, orthosis, gait, stroke, joint moment, motion analysis

\section{Background}

Hemiplegia is a typical symptom after the onset of stroke and causes a significant decrease in walking ability. ${ }^{1}$ The paralysis in patients with hemiplegia tends to be more severe distally, and a number of ankle-foot orthoses (AFOs) can be used to improve gait in these patients. ${ }^{2}$ It has been shown that AFOs improve the gait pattern and energy efficiency in patients with hemiplegia and improve spatiotemporal parameters, including walking speed, cadence, and step length. ${ }^{3-6}$ However, even though AFOs are easy to wear and remove, they can only help at a single joint, so their effect is localized. ${ }^{7}$ Therefore, researchers have sought ways of supporting the other joints in the lower extremities simultaneously to augment the effect of AFOs.

Hemiplegic patients are likely to experience hyperextension and buckling of the knee. Therefore, knee-ankle-foot orthoses are often used to assist the function of the knee joint. ${ }^{8}$ Further, several studies have reported on devices that support the hip joint in hemiplegic patients. Maguire et al demonstrated that an elasticated strap had an immediate effect of increasing hip abductor muscle activity in hemiplegic patients. ${ }^{9}$ In another study, Thijssen et al used an elastic strap to guide the major joints of the affected limb (ie, the hip, knee, and ankle) and reported both an immediate effect and 
an effect after familiarization for 3 weeks on the energy cost and spatiotemporal parameters of level walking. ${ }^{10}$ Further, Carda et al reported immediate improvement in scores on the 6-min and 10-m walk tests in patients wearing an orthosis designed to assist hip flexion. ${ }^{11}$

As described above, there are already some devices other than AFOs that can assist paretic lower limb joints in the hemiplegic patient. However, there is increasing emphasis on rehabilitation of the trunk in hemiplegic patients because trunk performance contributes to gait performance. ${ }^{12,13}$ Unfortunately, there has been little progress in development of trunk orthoses to supplement the function of the torso in patients with hemiplegia. Katsuhira et al developed a trunk orthosis with joints providing resistive force (TORF; Trunk Solution Co., Ltd, Tokyo, Japan) and investigated its effect on kinetic and kinematic parameters of the hemiplegic gait, reporting many effects in the paretic lower limb. ${ }^{14}$ However, in that study, the hemiplegic subjects walked without an AFO while wearing the TORF and no control group was included. If a combination of an AFO and a trunk orthosis could improve gait performance in hemiplegic patients, it might be useful as a rehabilitation intervention. In this study, a synergetic effect was defined as two different types of interventions providing a positive effect. A previous study reported that tibial nerve blockade and an AFO had a synergetic effect on hemiplegic gait, ${ }^{15}$ but the synergetic effect of two different orthotic strategies has not been studied.

The aim of this study was to compare the immediate effect on hemiplegic gait of wearing a trunk orthosis in addition to an AFO. We hypothesized that wearing a TORF as the trunk orthosis would stabilize the upper trunk and pelvis, thereby supplementing trunk function and improving hemiplegic gait performance.

\section{Methods}

\section{Features of the TORF}

The design and features of the TORF have been described previously. ${ }^{14}$ Briefly, a pelvic and upper support is positioned on the ilium and sternum, respectively, and the joints are connected with a silicon pad to an upper support made of stainless steel and to a pelvic support (Figure 1). The joints produce resistive force via extension springs, and a link mechanism translates the spring-generated tension into resistive force on the chest. Although anterior bending of the trunk is restricted by the resistive force, the upper support allows $40^{\circ}$ of movement in the anterior direction. However, there is no restriction of movement of the upper trunk in the mediolateral direction. While wearing the TORF, the upper support initially inclines backward to exert resistive force on the chest. The TORF has release mechanics produced by tension levers whereby resistive force is released by pulling the tension levers downward. Adjustable screws control the magnitude of spring-generated resistive force.

We previously reported that our prototype TORF could enhance the forward tilt of the pelvis and upright posture of the trunk, thereby increasing the flexion moment exerted by the abdominal muscles and decreasing the extension moment exerted by the back muscles during level walking in community-dwelling elderly people. ${ }^{16}$

\section{Patients}

The participants comprised 28 patients residing in Kanagawa Prefecture $(n=13)$ or Fukuoka Prefecture $(n=15)$ in Japan who were in the chronic phase of stroke. Patients in this phase of stroke were selected because the recovery curve plateaus and the gait performance reaches a steady state at $\sim 6$ months after onset. ${ }^{17}$ The inclusion criteria were a diagnosis of hemiparesis and the ability to walk at least $10 \mathrm{~m}$. The exclusion criteria were nonconcurrent pathology affecting the neuromuscular or central nervous system, communication problems, severe spasticity, and proprioceptive sensory impairment. We randomly allocated the 28 patients to a TORF group or a control group (Figure 2). One patient from Fukuoka was found to be ineligible for inclusion because of missing markers, leaving data for 27 patients (TORF group, $n=13$; control group, $\mathrm{n}=14$ ). Table 1 shows the participants' demographic and clinical characteristics. The study protocol was approved by the institutional ethics committees at the International University of Health and Welfare (14-Io-102) and Seiai Rehabilitation Hospital (14-1225) and followed the tenets of the Declaration of Helsinki. Written informed consent was obtained from all subjects prior to their participation in the study.

\section{Study protocol}

The participants were randomly allocated to the study groups by a statistician using a computerized random number generator. A physical therapist informed the participants of their allocation numbers. The study protocol has been published elsewhere. ${ }^{14}$ We recorded the gait data using a three-dimensional (3D) motion capture system consisting of 8 or 12 cameras (VICON Mx; VICON, Oxford, UK) and four or six force plates (AMTI, Watertown, MA, USA) arranged in two or three rows of two. The tests were performed at two separate institutions. The patient stepped onto the righthand force plates with the right foot and the left-hand force plates with the left foot. Thirty-nine reflective markers were attached using the marker model developed by Helen Hayes. An ankle-joint marker was placed on the mechanical joint 

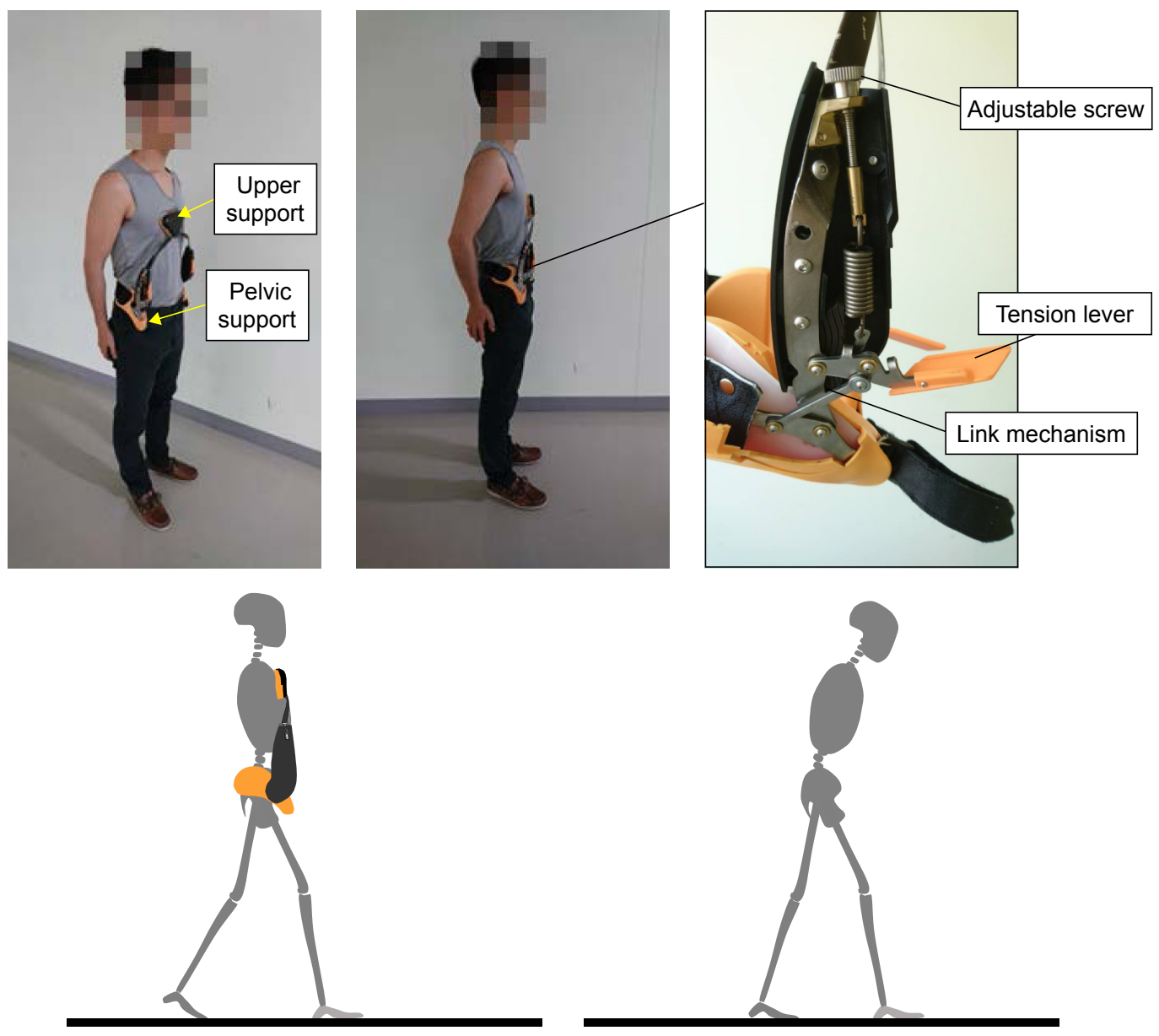

Figure I Trunk orthosis with joints providing resistive force.

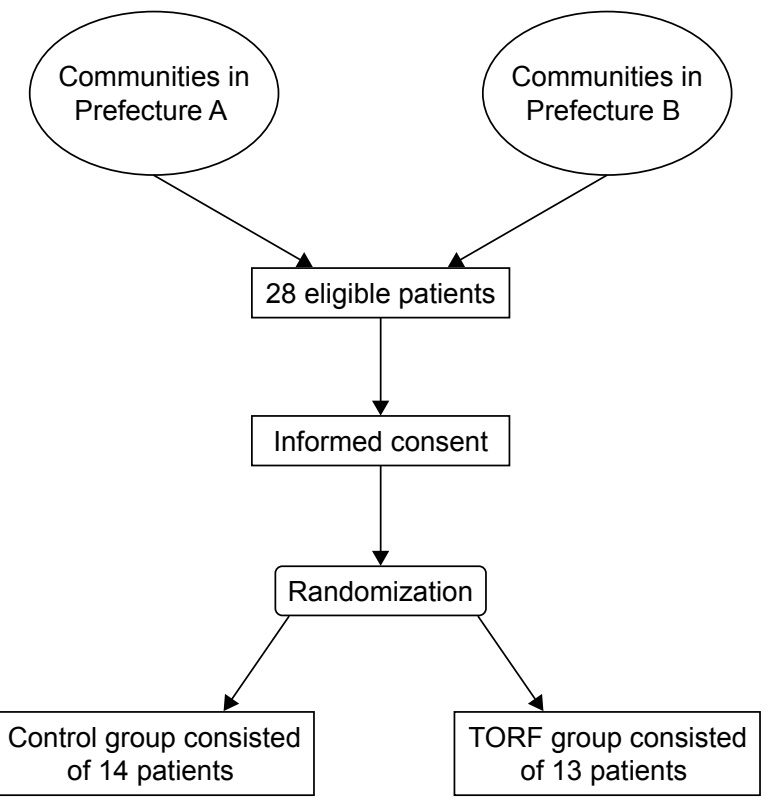

Figure 2 Flow of patients through the trial.

Note: One patient was excluded after measurements were taken because of missing markers.

Abbreviation: TORF, trunk orthosis with joints providing resistive force. of the AFO. Markers for the trunk and pelvis were placed on the participant's skin. An additional marker was placed on the center of the sacrum. Bilateral markers for the anterior superior iliac spine were removed to prevent interference when both trunk orthoses were worn but were reapplied on the posterior superior iliac spine bilaterally during walking and on the center of the sacrum during static standing when the trunk orthoses were not being worn. We measured the trajectories of the markers and the force plate data at a sampling frequency of $100 \mathrm{~Hz}$ in both laboratories. All patients were wearing an AFO (Gait Solution Design; Kawamura Gishi, Daito, Japan) and used canes as needed during all measurement sessions. Patients in the control group wore a lumbosacral orthosis (Damen Corset; Pacific Supply, Osaka, Japan) and those in the TORF group wore a TORF. First, we measured gait when the patient was wearing an AFO without any trunk orthosis at the patient's selected speed and repeated these measurements three times. Next, we measured gait when the patient was wearing an AFO with their allocated trunk orthosis (TORF or corset). 
Table I Patient demographic and clinical characteristics

\begin{tabular}{|c|c|c|c|c|c|c|c|c|c|}
\hline $\begin{array}{l}\text { Patient } \\
\text { ID }\end{array}$ & $\begin{array}{l}\text { Age } \\
\text { (years) }\end{array}$ & Sex & Height (cm) & Weight (kg) & Diagnosis & $\begin{array}{l}\text { Paretic } \\
\text { side }\end{array}$ & $\begin{array}{l}\text { BrS of lower } \\
\text { extremities }\end{array}$ & $\begin{array}{l}\text { Time since } \\
\text { onset (years) }\end{array}$ & Walking aid \\
\hline \multicolumn{10}{|c|}{ TORF group } \\
\hline A & 75 & $\mathrm{~F}$ & 159.0 & 68.0 & Cerebral infarction & $\mathrm{R}$ & $\mathrm{VI}$ & 5 & None \\
\hline B & 63 & M & 169.0 & 78.0 & Cerebral hemorrhage & $\mathrm{R}$ & III & 12 & None \\
\hline C & 76 & M & 161.0 & 66.3 & Cerebral infarction & $\mathrm{R}$ & $\mathrm{V}$ & 10 & None \\
\hline $\mathrm{D}$ & 66 & M & 169.0 & 64.9 & Cerebral hemorrhage & $\mathrm{R}$ & IV & 11 & None \\
\hline $\mathrm{E}$ & 77 & $M$ & 168.0 & 76.7 & Cerebral hemorrhage & $\mathrm{R}$ & $\vee$ & 15 & None \\
\hline $\mathrm{F}$ & 65 & $M$ & 173.0 & 53.8 & Cerebral hemorrhage & $\mathrm{R}$ & $\mathrm{V}$ & 2 & None \\
\hline G & 73 & M & 165.0 & 71.2 & Cerebral hemorrhage & $\mathrm{L}$ & IV & 14 & T-cane \\
\hline $\mathrm{H}$ & 78 & M & 163.0 & 62.3 & Cerebral hemorrhage & $\mathrm{L}$ & VI & 26 & None \\
\hline I & 78 & $\mathrm{~F}$ & 148.0 & 40.2 & Cerebral hemorrhage & $\mathrm{L}$ & IV & 16 & None \\
\hline$J$ & 71 & $M$ & 162.0 & 72.0 & Cerebral infarction & $\mathrm{L}$ & $\mathrm{V}$ & 5 & None \\
\hline $\mathrm{K}$ & 66 & $\mathrm{~F}$ & 147.5 & 58.2 & Cerebral hemorrhage & $\mathrm{L}$ & IV & 9 & T-cane \\
\hline $\mathrm{L}$ & 78 & $M$ & 160.0 & 49.8 & Cerebral infarction & $\mathrm{L}$ & IV & 4 & T-cane \\
\hline $\mathrm{N}$ & 57 & M & 169.0 & 57.0 & Cerebral hemorrhage & $\mathrm{L}$ & IV & 5 & T-cane \\
\hline Mean & 71.0 & & 162.6 & 63.0 & & & & 10.3 & \\
\hline SD & 6.92 & & 7.80 & 11.0 & & & & 6.54 & \\
\hline \multicolumn{10}{|c|}{ Control group } \\
\hline M & 68 & $M$ & 174.0 & 77.2 & Cerebral infarction & $\mathrm{R}$ & VI & 5 & None \\
\hline O & 73 & $\mathrm{~F}$ & 152.0 & 51.8 & Cerebral infarction & $\mathrm{R}$ & VI & 10 & T-cane \\
\hline$P$ & 57 & $M$ & 173.0 & 89.9 & Cerebral hemorrhage & $\mathrm{R}$ & IV & 2 & T-cane \\
\hline $\mathrm{Q}$ & 71 & M & 173.0 & 60.0 & Cerebral infarction & $\mathrm{R}$ & $\vee$ & 11 & None \\
\hline $\mathrm{R}$ & 62 & $\mathrm{~F}$ & 151.0 & 47.0 & Cerebral hemorrhage & $\mathrm{R}$ & $\mathrm{V}$ & 3 & None \\
\hline$S$ & 74 & $M$ & 163.5 & 57.8 & Cerebral hemorrhage & $\mathrm{L}$ & III & 14 & T-cane \\
\hline $\mathrm{T}$ & 75 & M & 163.0 & 57.6 & Cerebral hemorrhage & $\mathrm{L}$ & $\mathrm{V}$ & 11 & None \\
\hline$U$ & 84 & $M$ & 158.0 & 58.4 & Cerebral infarction & $\mathrm{L}$ & IV & 20 & T-cane \\
\hline V & 66 & $\mathrm{~F}$ & 154.0 & 63.2 & Cerebral hemorrhage & L & IV & 4 & T-cane \\
\hline W & 62 & M & 164.0 & 45.0 & Cerebral hemorrhage & $\mathrm{L}$ & IV & 1 & T-cane \\
\hline$x$ & 73 & $\mathrm{~F}$ & 147.0 & 45.5 & Cerebral infarction & $\mathrm{L}$ & $\mathrm{V}$ & 2 & None \\
\hline$Y$ & 68 & $\mathrm{~F}$ & 155.0 & 41.0 & Cerebral infarction & L & $\vee$ & 3 & None \\
\hline $\mathrm{Z}$ & 80 & $M$ & 161.0 & 55.0 & Cerebral hemorrhage & L & VI & 2 & None \\
\hline $\mathrm{A} 2$ & 66 & M & 159.0 & 50.0 & Cerebral hemorrhage & $\mathrm{L}$ & $\vee$ & 1 & T-cane \\
\hline Mean & 69.9 & & 160.5 & 57.1 & & & & 6.4 & \\
\hline SD & 7.3 & & 8.5 & 13.2 & & & & 5.85 & \\
\hline
\end{tabular}

Abbreviations: BrS, Brunnstrom recovery stage; TORF, trunk orthosis with joints providing resistive force.

Before starting measurements, all subjects practiced walking on the floor of the laboratory at a self-selected speed for $10 \mathrm{~min}$ to become accustomed to wearing their AFO and allocated trunk orthosis while holding a break. Before measuring gait, we set the magnitude of the resistive force to $20 \mathrm{~N}$ during static standing while wearing the TORF based on the results of the preliminary experiments. We measured the adjustments to the resistive force with a strain gauge embedded in the upper support of the TORF and transferred the measurement to a personal computer using Bluetooth technology. The pressure between the lumbosacral orthosis and the abdomen was set to $10 \mathrm{mmHg}$ under all measurement conditions. ${ }^{18}$ Measurements were recorded for all patients in both groups with and without their trunk orthosis (TORF or lumbosacral corset).

\section{Data processing}

We processed the physical coordinates and ground reaction force (GRF) data using a 6- and 18-Hz low-pass filter, respectively. The data were analyzed using Visual 3D version 6 analytical software (C-motion, Inc., Germantown, MD, USA). The link segment model consisted of 13 segments (head, trunk, pelvis, upper arms, forearms, thighs, shanks, and feet). We calculated joint kinematics and kinetics according to Winter's technique. ${ }^{19}$ From these data, we detected the spatiotemporal parameters and the peak values for triaxial joint angles, joint moments, and GRF per gait cycle in each patient under each condition; the reason for doing this was that a previous study investigating the effects of the TORF on hemiplegic gait used the same parameters ${ }^{14}$ and another study by Yamamoto et al used the same AFO and reported on the same parameters. ${ }^{20}$ However, those studies reported kinetic and kinematic parameters mainly in the sagittal plane and neither reported on the alignment of the pelvis and trunk. A further study had investigated the effect of the TORF on alignment in healthy elderly subjects. Therefore, we decided to analyze GRF, triaxial lower joint kinetic and 
kinematics, and triaxial movements of the trunk and pelvis. Joint moments and GRF were normalized by body mass. The peak values for paretic kinetic and kinematic parameters at the time of 1) initial contact, 2) loading response (LR), 3) pre-swing (PSw), 4) single stance (SS), and 5) swing were extracted for analysis. We defined these phases using the vertical component of GRF.

\section{Statistical analysis}

Gait parameters were determined and averaged for at least two of three measured trials without missing markers under each of the two experimental conditions. The spatiotemporal, kinetic, and kinematic parameters were not normally distributed according to the Shapiro-Wilk test, so a nonparametric statistical analysis was performed. First, the Wilcoxon signed-rank test was used to identify any significant differences between wearing and not wearing a trunk orthosis. When a significant difference was found, further comparisons were made between baseline and wearing a trunk orthosis using the Mann-Whitney $U$-test. A $p$-value of 0.05 was considered to be statistically significant. All statistical analyses were performed using SPSS for Windows version 21 software (IBM Corp., Armonk, NY, USA).

\section{Results}

No significant difference in patient height ( $p=0.451)$, weight ( $p=0.133)$, or age $(p=0.576)$ was found between the two groups (Table 1).

\section{Comparison with and without a trunk orthosis}

There were statistically significant differences in values for all spatiotemporal gait parameters between when patients were wearing and not wearing a trunk orthosis (Table 2). Walking speed and number of paretic steps per minute increased significantly, and the number of paretic steps decreased significantly in the TORF condition but not in the corset condition. Significant changes in spatiotemporal parameters were observed only on the paretic side.

Some kinetic and kinematic parameters showed significant changes under the TORF condition but not under the corset condition (Table 3 ). In addition, there were significant differences in angles at all joints of the lower extremities, pelvis, and thorax and GRF values between the TORF condition and the non-TORF condition. In the TORF group, the peak mediolateral component of the GRF during the LR phase and the vertical component of the GRF in the PSw phase increased significantly in the paretic limb when the trunk orthosis was worn compared to those when it was not. The peak ankle plantar flexion moment during the SS phase and the dorsiflexion moment during the swing phase increased significantly in the TORF condition when compared with the non-TORF condition. The peak knee abduction moment during the PSw phase also increased significantly in the TORF condition when compared with the non-TORF condition. The peak hip extension moment in the swing phase and the extension angle in the SS and PSw phases also increased significantly in the TORF condition when compared with the non-TORF condition.

The angles of the pelvis and thorax during one gait cycle of the paretic limb are shown in Table 4. The peak pelvic tilt angle in the LR phase increased significantly in the TORF condition when compared with the non-TORF condition. Peak thorax bending angles over one gait cycle in the paretic limb decreased significantly in both the TORF condition and the corset condition, except during the LR phase. The peak thorax lateral bending angle during the SS, PSw, and swing phases decreased significantly in the TORF condition when compared with the non-TORF condition but not in the corset condition when compared with the non-corset condition.

Table 2 Comparison of spatiotemporal parameters between conditions with and without trunk orthoses

\begin{tabular}{|c|c|c|c|c|c|c|c|}
\hline & \multicolumn{3}{|c|}{ Non-TORF condition } & \multicolumn{3}{|c|}{ TORF condition } & \multirow[t]{2}{*}{$p$-value } \\
\hline & Median & $25 \%-7$ & & Median & $25 \%-$ & & \\
\hline \multicolumn{8}{|l|}{ Spatiotemporal parameter } \\
\hline Walking speed $(\mathrm{m} / \mathrm{s})$ & 0.532 & 0.387 & 0.596 & 0.582 & 0.381 & 0.659 & 0.013 \\
\hline Paretic step time (s) & 0.790 & 0.740 & 0.962 & 0.763 & 0.670 & 0.923 & 0.048 \\
\hline \multirow[t]{3}{*}{ Paretic steps/min (n) } & 76.0 & 62.6 & 81.6 & 78.6 & 65.1 & 89.8 & 0.022 \\
\hline & \multicolumn{3}{|c|}{ Non-corset condition } & \multicolumn{3}{|c|}{ Corset condition } & \multirow[t]{2}{*}{$p$-value } \\
\hline & Median & $25 \%-7$ & & Median & $25 \%-$ & & \\
\hline \multicolumn{8}{|l|}{ Spatiotemporal parameter } \\
\hline Walking speed $(\mathrm{m} / \mathrm{s})$ & 0.446 & 0.366 & 0.578 & 0.458 & 0.373 & 0.569 & 0.626 \\
\hline Paretic step time (s) & 0.71 & 0.656 & 0.975 & 0.755 & 0.673 & 0.967 & 0.542 \\
\hline Paretic steps/min (n) & 77.2 & 50.0 & 89.7 & 79.5 & 62.3 & 89.2 & 0.855 \\
\hline
\end{tabular}

Note: Bold figures indicate statistically significant $p<0.05$. 
Table 3 Comparison of kinetic and kinematic paretic lower limb parameters between conditions with and without trunk orthoses

\begin{tabular}{|c|c|c|c|c|c|c|c|}
\hline & \multicolumn{3}{|c|}{ Non-TORF condition } & \multicolumn{3}{|c|}{ TORF condition } & \multirow[t]{2}{*}{$p$-value } \\
\hline & Median & $25 \%-7$ & & Median & $25 \%-$ & & \\
\hline \multicolumn{8}{|l|}{ Ground reaction force } \\
\hline Peak mediolateral component during LR (N/kg) & 0.071 & 0.066 & 0.090 & 0.080 & 0.069 & 0.093 & 0.023 \\
\hline Peak vertical component during PSw (N/kg) & 0.944 & 0.893 & 0.966 & 0.955 & 0.924 & 0.995 & 0.048 \\
\hline \multicolumn{8}{|l|}{ Ankle } \\
\hline Peak plantar flexion moment during SS ( $\mathrm{N} \mathrm{m/kg)}$ & 0.732 & 0.644 & 0.839 & 0.784 & 0.630 & 0.980 & 0.013 \\
\hline Peak dorsiflexion moment during swing $(\mathrm{N} \mathrm{m} / \mathrm{kg})$ & 0.021 & 0.020 & 0.024 & 0.022 & 0.021 & 0.027 & 0.013 \\
\hline \multicolumn{8}{|l|}{ Knee } \\
\hline Peak knee abduction moment during PSw ( $\mathrm{N} \mathrm{m/kg)}$ & 0.324 & 0.170 & 0.373 & 0.346 & 0.220 & 0.382 & 0.006 \\
\hline \multicolumn{8}{|l|}{ Hip } \\
\hline Peak extension moment in swing ( $\mathrm{N} \mathrm{m/kg)}$ & 0.115 & 0.079 & 0.134 & 0.118 & 0.049 & 0.196 & 0.048 \\
\hline Peak extension angle in SS $\left(^{\circ}\right)$ & 4.70 & 1.08 & 10.34 & 5.57 & 1.96 & 14.45 & 0.027 \\
\hline \multirow[t]{3}{*}{ Peak extension angle in PSw $\left(^{\circ}\right)$} & 8.68 & 4.23 & 10.78 & 8.85 & 4.80 & 12.69 & 0.006 \\
\hline & \multicolumn{3}{|c|}{ Non-corset condition } & \multicolumn{3}{|c|}{ Corset condition } & \multirow[t]{2}{*}{$p$-value } \\
\hline & Median & $25 \%-7$ & & Median & $25 \%-$ & & \\
\hline \multicolumn{8}{|l|}{ Ground reaction force } \\
\hline Peak mediolateral component during LR (N/kg) & 0.073 & 0.058 & 0.087 & 0.080 & 0.060 & 0.091 & 0.583 \\
\hline Peak vertical component during PSw (N/kg) & 0.846 & 0.802 & 0.933 & 0.882 & 0.838 & 0.937 & 0.358 \\
\hline \multicolumn{8}{|l|}{ Ankle } \\
\hline Peak plantar flexion moment during SS ( $\mathrm{N} \mathrm{m/kg)}$ & 0.628 & $0.47 \mathrm{I}$ & 0.872 & 0.610 & 0.493 & 0.840 & 0.963 \\
\hline Peak dorsiflexion moment during swing ( $\mathrm{N} \mathrm{m/kg)}$ & 0.019 & 0.016 & 0.022 & 0.021 & 0.017 & 0.022 & 1.000 \\
\hline \multicolumn{8}{|l|}{ Knee } \\
\hline Peak knee abduction moment during PSw (N m/kg) & 0.276 & 0.179 & 0.317 & 0.287 & 0.237 & 0.349 & 0.626 \\
\hline \multicolumn{8}{|l|}{ Hip } \\
\hline Peak extension moment in swing ( $\mathrm{N} \mathrm{m/kg)}$ & 0.100 & 0.020 & 0.162 & 0.085 & 0.037 & 0.161 & 0.952 \\
\hline Peak extension angle in SS $\left(^{\circ}\right)$ & 4.40 & 0.55 & 6.51 & 4.13 & 0.50 & 8.36 & 0.626 \\
\hline Peak extension angle in PSw $\left(^{\circ}\right)$ & 6.25 & 4.18 & 8.94 & 6.89 & 11.81 & 4.55 & 0.326 \\
\hline
\end{tabular}

Note: Bold figures indicate statistically significant $p<0.05$.

Abbreviations: LR, loading response; PSw, pre-swing; SS, single stance; TORF, trunk orthosis with joints providing resistive force.

\section{Comparison of changes from baseline between the TORF and corset groups}

All changes in parameters from baseline are compared between the TORF and corset groups in Table 5. In the TORF condition, we observed significant increases in walking speed, paretic steps per minute, peak ankle plantar flexion moment during the SS phase, and dorsiflexion moment during the swing phase, as well as a significant decrease in paretic step time. The ankle plantar flexion moment increased at the end of the SS phase, which is the terminal stance phase shown in Figure 3.

\section{Discussion}

Our results demonstrate that wearing a TORF and an AFO at the same time can improve gait performance in hemiplegic patients by significantly increasing their gait speed and ankle-joint plantar flexion moment toward the end of the SS phase to a greater extent than is possible wearing a corset and an AFO. Further, the TORF significantly modified the malalignment commonly seen in hemiplegic patients.

\section{Changes from baseline}

We hypothesized that wearing both a TORF and a corset would augment gait performance by improving trunk function. However, subjects in the TORF group showed greater functional improvement in their hemiplegic lower limb because of the stabilizing effect on the upper trunk of the resistive force applied to the chest. In addition, significant changes from baseline were observed only in the TORF condition. The spatiotemporal parameters of gait speed and number of paretic steps per minute significantly increased and the paretic step time significantly decreased in the TORF condition. A previous study investigating the effect of a TORF on hemiplegic patient did not show significant improvement of gait speed compared with that before wearing a TORF ${ }^{14}$ because no AFO was applied. It is reported that AFOs are able to facilitate weight-bearing on the paretic limb and increase stability during stance in stroke patients, ${ }^{21}$ and thus might contribute to a decrease in the compensatory trunk movements seen in patients with a hemiplegic gait. ${ }^{22}$ The synergistic effect of wearing a TORF to stabilize the trunk and 
Table 4 Comparison of pelvis and thorax angles between conditions with and without trunk orthoses

\begin{tabular}{|c|c|c|c|c|c|c|c|}
\hline & \multicolumn{3}{|c|}{ Non-TORF condition } & \multicolumn{3}{|c|}{ TORF condition } & \multirow[t]{2}{*}{$p$-value } \\
\hline & Median & $25 \%-7$ & & Median & $25 \%-7$ & & \\
\hline \multicolumn{8}{|c|}{ Pelvis angle forward tilt angle $\left({ }^{\circ}\right)$} \\
\hline Peak during LR & 0.84 & -3.60 & 3.22 & -1.40 & -6.95 & 0.22 & 0.023 \\
\hline Peak during swing & -0.09 & -4.92 & 2.90 & -2.13 & -6.50 & 1.80 & 0.048 \\
\hline \multicolumn{8}{|c|}{ Thorax bending angle $\left({ }^{\circ}\right)$ : forward+ } \\
\hline Peak during LR & 3.59 & -0.08 & 7.90 & -0.28 & -2.02 & 5.99 & 0.003 \\
\hline Peak during SS & 5.62 & 2.31 & 10.62 & 1.60 & 0.22 & 8.02 & 0.002 \\
\hline Peak during PSw & 3.80 & -0.15 & 8.84 & 0.28 & -3.30 & 6.34 & 0.005 \\
\hline Peak during swing & 6.76 & 4.43 & 12.06 & 2.32 & -0.74 & 8.29 & 0.002 \\
\hline \multicolumn{8}{|c|}{ Thorax lateral bending angle $\left({ }^{\circ}\right)$ : paretic side +} \\
\hline Peak during SS & 4.27 & -2.24 & 5.65 & 3.57 & -2.39 & 6.34 & 0.033 \\
\hline Peak during PSw & 2.07 & -3.06 & 4.97 & 1.49 & -3.09 & 3.52 & 0.017 \\
\hline \multirow[t]{3}{*}{ Peak during swing } & 1.78 & -3.20 & 3.49 & 1.19 & -3.91 & 2.35 & 0.040 \\
\hline & \multicolumn{3}{|c|}{ Non-corset condition } & \multicolumn{3}{|c|}{ Corset condition } & \multirow[t]{2}{*}{$p$-value } \\
\hline & Median & $25 \%-7$ & & Median & $25 \%-7$ & & \\
\hline \multicolumn{8}{|c|}{ Pelvis angle forward tilt angle $\left({ }^{\circ}\right)$} \\
\hline Peak during LR & -1.29 & -4.74 & 0.26 & -1.83 & -4.78 & 3.99 & 0.715 \\
\hline Peak during swing & -1.87 & -3.32 & 0.19 & -1.47 & -4.18 & 3.13 & 0.426 \\
\hline \multicolumn{8}{|c|}{ Thorax bending angle $\left({ }^{\circ}\right)$ : forward +} \\
\hline Peak during LR & 4.37 & 1.50 & 12.89 & 3.42 & 0.61 & 11.13 & 0.091 \\
\hline Peak during SS & 5.62 & 3.54 & 11.95 & 6.81 & 3.09 & 12.89 & 0.025 \\
\hline Peak during Psw & 7.85 & 4.47 & 16.51 & 6.51 & 4.89 & 14.72 & 0.025 \\
\hline Peak during swing & 9.21 & 4.16 & 16.08 & 7.68 & 5.09 & 14.58 & 0.025 \\
\hline \multicolumn{8}{|c|}{ Thorax lateral bending angle $\left({ }^{\circ}\right)$ : paretic side +} \\
\hline Peak during SS & 2.15 & -0.34 & 4.54 & 1.37 & -0.22 & 4.23 & 0.583 \\
\hline Peak during PSw & -0.97 & -3.67 & 2.28 & -0.27 & -2.77 & 1.78 & 1.000 \\
\hline Peak during swing & -1.66 & -4.63 & 0.99 & -1.02 & -3.64 & 1.90 & 0.903 \\
\hline
\end{tabular}

Notes: Bold figures indicate statistically significant $p<0.05$.

Abbreviations: LR, loading response; PSw, pre-swing; SS, single stance; TORF, trunk orthosis with joints providing resistive force.

an AFO might contribute to this decrease in compensatory trunk movements, leading to significant changes in spatiotemporal parameters.

The most interesting change in the TORF condition was the significant increase in ankle plantar flexion moment during the SS phase. Nadeau et al reported that weakness of the plantar flexors should be considered as a factor limiting gait speed ${ }^{23}$ Further, Yamamoto et al emphasized the importance of the plantar flexion moment as an indicator of the ankle rocker function relating to forward progression of the center of pressure in hemiplegic patients..$^{20}$ Yamamoto et al used an AFO similar to the one used in the present study and reported that their device increased the ankle plantar flexion moment after gait training for 3 weeks but not immediately after application. ${ }^{20}$ In the present study, the ankle plantar flexion moment increased after only 10 min of familiarization with wearing the TORF and AFO. Moreover, this significant increase in ankle plantar flexion moment could have led to significant changes in spatiotemporal parameters, including gait speed. Accordingly, using a TORF might enhance the effect of gait training wearing the AFO. Further, the slight increase in ankle dorsiflexion moment during the swing phase, which helps to prevent foot drop, was observed in the TORF condition; this increase in moment might have been caused by an increase in inertial force as a result of a faster gait speed, but this was too small to determine if there was an effect on gait or not.

\section{Differences in gait parameters with and without a trunk orthosis}

Parameters that showed a significant change from baseline also showed a significant difference between the TORF and non-TORF conditions. These differences may help to explain the beneficial effect of a TORF in hemiplegic stroke patients.

More significant differences were observed between the TORF and non-TORF conditions than between the corset and non-corset conditions. No significant differences were observed between the corset and non-corset conditions except for a significant decrease in the trunk 
Table 5 Comparison of changes from baseline between the TORF and corset groups

\begin{tabular}{|c|c|c|c|c|c|c|c|}
\hline & \multicolumn{3}{|c|}{$\begin{array}{l}\text { TORF group: change from } \\
\text { baseline }\end{array}$} & \multicolumn{3}{|c|}{$\begin{array}{l}\text { Corset group: change from } \\
\text { baseline }\end{array}$} & \multirow[t]{2}{*}{$p$-value } \\
\hline & Median & $25 \%-75 \%$ & & Median & $25 \%-75$ & & \\
\hline \multicolumn{8}{|l|}{ Spatiotemporal parameter } \\
\hline Walking speed $(\mathrm{m} / \mathrm{s})$ & 0.023 & 0.005 & 0.066 & -0.002 & -0.024 & 0.01 & 0.015 \\
\hline Paretic step time (s) & -0.030 & -0.038 & -0.020 & 0.008 & -0.019 & 0.048 & 0.047 \\
\hline Paretic steps/min, n & 2.50 & 0.98 & 3.98 & -0.60 & -2.005 & 1.881 & 0.037 \\
\hline \multicolumn{8}{|l|}{ Ground reaction force } \\
\hline Peak mediolateral component during LR (N/kg) & 0.005 & 0.002 & 0.011 & 0.004 & -0.009 & 0.012 & 0.594 \\
\hline Peak vertical component during PSw (N/kg) & 0.025 & 0.011 & 0.036 & 0.005 & -0.011 & 0.038 & 0.734 \\
\hline \multicolumn{8}{|l|}{ Ankle } \\
\hline Peak plantar flexion moment during SS (N m/kg) & 0.073 & -0.006 & 0.101 & -0.037 & -0.090 & 0.014 & 0.023 \\
\hline Peak dorsiflexion moment during swing ( $\mathrm{N} \mathrm{m} / \mathrm{kg})$ & 0.001 & 0.000 & 0.002 & 0.000 & 0.000 & 0.001 & 0.042 \\
\hline \multicolumn{8}{|l|}{ Knee } \\
\hline Peak knee abduction moment during PSw ( $\mathrm{N} \mathrm{m/kg)}$ & 0.023 & 0.004 & 0.040 & -0.002 & -0.018 & 0.033 & 0.133 \\
\hline \multicolumn{8}{|l|}{ Hip } \\
\hline Peak extension moment in swing $(\mathrm{N} \mathrm{m} / \mathrm{kg})$ & 0.025 & -0.003 & 0.062 & -0.005 & -0.033 & 0.031 & 0.146 \\
\hline Peak extension angle in SS $\left(^{\circ}\right)$ & 1.34 & 0.87 & 2.05 & 0.24 & -0.96 & 1.43 & 0.190 \\
\hline Peak extension angle in PSw $\left({ }^{\circ}\right)$ & 1.16 & 0.39 & 1.86 & 0.34 & -0.34 & 1.07 & 0.264 \\
\hline \multicolumn{8}{|l|}{ Pelvis tilt angle $\left({ }^{\circ}\right)$ : backward +} \\
\hline Peak during LR & 2.13 & 0.14 & 3.61 & 0.73 & -2.12 & 1.43 & 0.065 \\
\hline \multicolumn{8}{|l|}{ Thorax bending angle $\left({ }^{\circ}\right)$ : forward +} \\
\hline Peak during LR & -2.14 & -3.22 & -1.45 & -1.38 & -2.27 & -1.24 & $0.08 \mathrm{I}$ \\
\hline Peak during SS & -2.28 & -4.28 & -1.16 & -2.20 & -2.56 & -1.55 & 0.438 \\
\hline Peak during PSw & -2.66 & -3.52 & -0.75 & -1.57 & -2.18 & -0.65 & 0.244 \\
\hline Peak during swing & -3.54 & -4.44 & -2.29 & -2.06 & -2.54 & -0.49 & 0.052 \\
\hline \multicolumn{8}{|l|}{ Thorax lateral bending angle $\left({ }^{\circ}\right)$ : paretic side+ } \\
\hline Peak during SS & -0.70 & -1.08 & -0.13 & 0.10 & -0.92 & 1.33 & 0.133 \\
\hline Peak during PSw & -0.43 & -1.11 & -0.12 & -0.23 & -0.95 & 1.60 & 0.286 \\
\hline Peak during swing & -0.64 & -1.29 & 0.00 & -0.03 & -0.77 & 0.78 & 0.109 \\
\hline
\end{tabular}

Notes: Bold figures indicate statistically significant $p<0.05$.

Abbreviations: LR, loading response; PSw, pre-swing; SS, single stance; TORF, trunk orthosis with joints providing resistive force.

forward bending angle in the corset condition. The trunk forward bending and mediolateral bending angles were also significantly decreased in the TORF condition when compared with the non-TORF condition. A systematic review of trunk movement in patients with a hemiplegic gait after stroke reported that gait performance was related to trunk movement not only in the sagittal plane but also in the frontal plane. ${ }^{22} \mathrm{~A}$ decrease in trunk movement in both planes while wearing a TORF could have contributed to the improvement in gait parameters. Interestingly, the TORF restricts
A

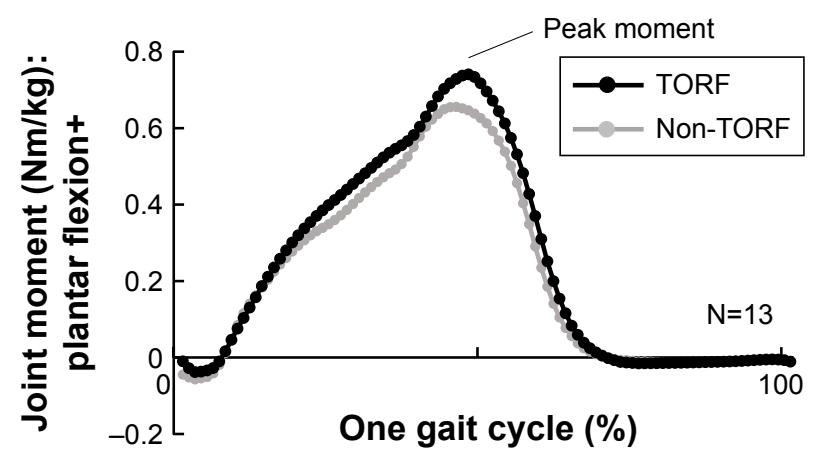

B

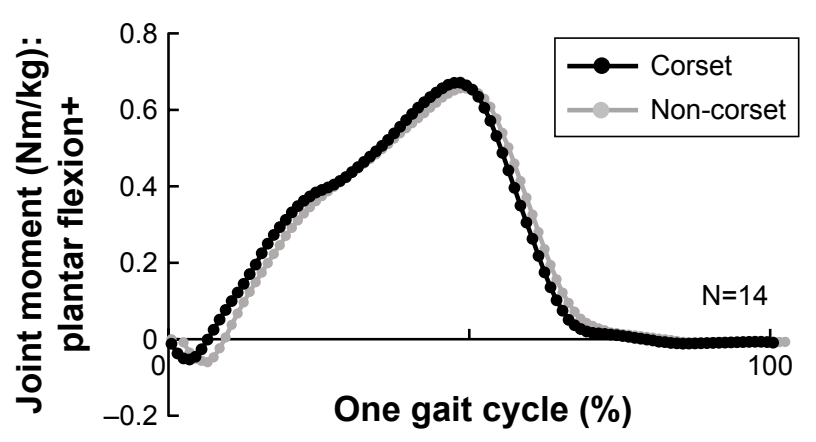

Figure 3 Comparison of ankle-joint moment between the TORF and non-TORF conditions and between the corset and non-corset conditions. Notes: (A) TORF group. (B) Corset group.

Abbreviation: TORF, trunk orthosis with joints providing resistive force. 


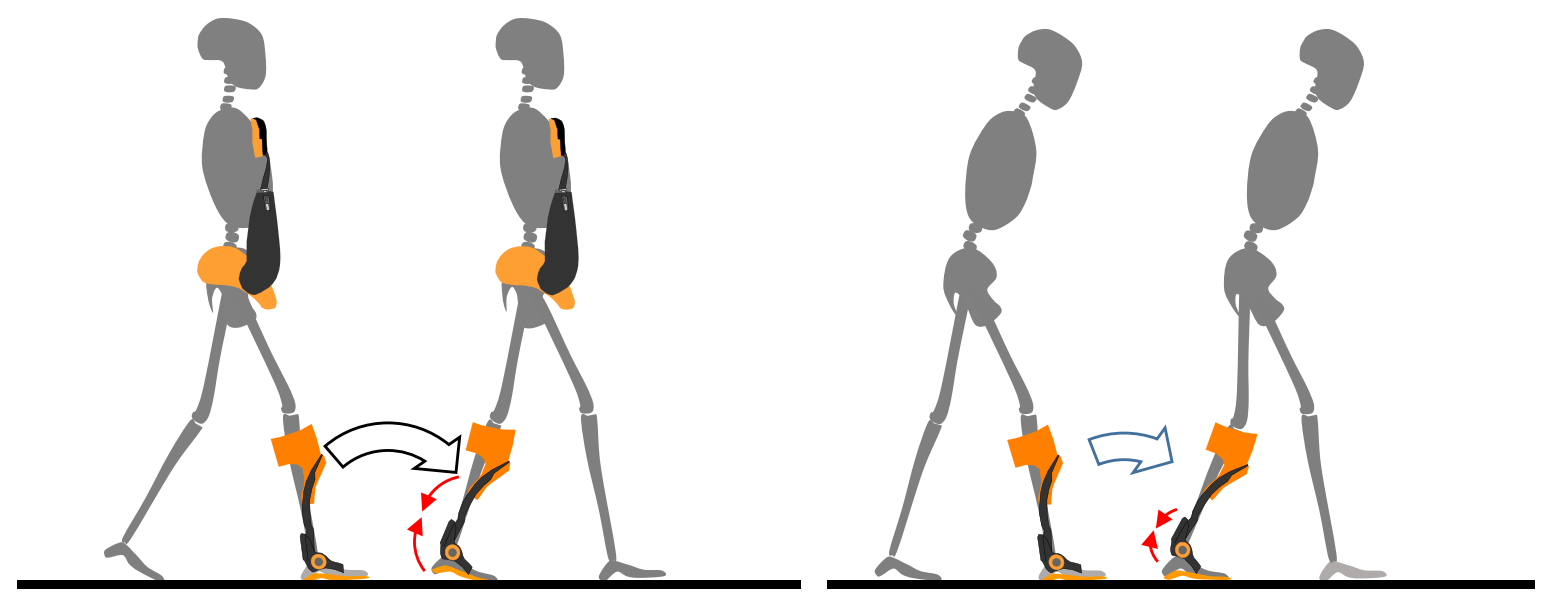

Figure 4 Synergetic effect of the trunk orthosis with joints providing resistive force and an ankle-foot orthosis on rocker function in subjects with a hemiplegic gait.

only forward but not mediolateral bending of the thorax with resistive force. Modified alignment in the sagittal plane and activation of the abdominal muscles by resistive force on the chest might have contributed to modified alignment even in the frontal plane. However, there was a significant increase in the mediolateral component of the GRF during the LR phase and in the internal abduction moment of the knee during the PSw phase. These increases might be caused by the restricted movement of the mediolateral trunk. An increase in the mediolateral component of the GRF provides stability in the same direction. However, it is well known that an increase in knee abduction moment is a cause of osteoporosis in the knee, ${ }^{24}$ so consideration might need to be given to increasing the load on the paretic knee joint while wearing a TORF.

There was no significant change in the trunk bending angle during the LR phase or in the pelvic tilt angle over one gait cycle in the corset condition. In contrast, there was a significant change in the trunk bending angle over one gait cycle and in the pelvic tilt angle during the LR phase in the TORF condition. Further, a significant change in hip extension angle in the SS and PSw phases was only observed in the TORF condition. These differences might be key to understanding the kinetic and kinematic differences observed between wearing the TORF and wearing a corset. As shown in Figure 4, an upright posture with a pelvic forward tilt is important when rotating the body using the ankle joint as the fulcrum from the LR phase to the terminal stance phase, which constitutes the second rocker phase, ${ }^{25}$ suggesting that this movement is accompanied by extension of the hip joint. Assisting the ankle joint using an AFO could be needed to achieve this movement, although a previous study designed to confirm the effect of the TORF in hemiplegic patients did not apply any AFO and did not report an increase in ankle plantar flexion moment or gait speed. ${ }^{14}$ In contrast, this moment increased significantly during the SS phase and gait speed also increased significantly because of the upright posture with pelvic forward tilt during the LR phase in the TORF condition. The TORF might enable an effective second rocker phase to be achieved easily using the ankle-joint moment. Use of a TORF and an AFO might not only assist the trunk and pelvis but also the ankle joint, so it might be a good combination therapy for improving gait in hemiplegic patients.

This study has several limitations. First, we only investigated the immediate effects of wearing a TORF on hemiplegic gait, so further research is needed to confirm the longer term effects after familiarization with the device. Second, there was some variation in the severity of hemiplegia in our study population, with some patients needing to use a cane while walking but not others. Further research is needed to determine whether use of a cane in addition to wearing a TORF and AFO has further synergetic effects in hemiplegic stroke patients. Third, we used only one type of AFO, so the synergistic effects that might exist for other combinations of AFOs and the TORF are as yet unknown. Finally, although this study underscores the importance of a systemic approach using different types of orthoses, more studies are required to confirm whether this strategy has a beneficial effect on motor control and coordination at the systemic level.

\section{Conclusion}

We have demonstrated that a TORF can enhance the effects of an AFO and that the synergistic effects of these devices can improve gait performance in hemiplegic patients by significantly increasing their gait speed and ankle-joint 
plantar flexion moment toward the end of the SS phase. Further, the TORF significantly modified the malalignment commonly seen in hemiplegic patients. We plan to conduct randomized controlled trials in the future to confirm the effect of continuous use of the TORF for gait training.

\section{Acknowledgments}

This study was supported by KAKENHI Grants-in-Aid (Nos 23700619 and 16K01516) from the Japan Society for the Promotion of Science. The funding source had no role in study design, data collection, and data analysis.

\section{Disclosure}

Junji Katsuhira and Ko Matsudaira are directors of Trunk Solution Co., Ltd. The authors report no other conflicts of interest in this work.

\section{References}

1. Belda-Lois JM, Mena-del Horno S, Bermejo-Bosch I, et al. Rehabilitation of gait after stroke: a review towards a top-down approach. J Neuroeng Rehabil. 2011;8(1):66.

2. Leung J, Moseley A. Impact of ankle-foot orthoses on gait and leg muscle activity in adults with hemiplegia: systematic literature review. Physiotherapy. 2003;89(1):39-55.

3. Mulroy SJ, Eberly VJ, Gronely JK, Weiss W, Newsam CJ. Effect of AFO design on walking after stroke: impact of ankle plantar flexion contracture. Prosthet Orthot Int. 2010;34(3):277-292.

4. Desloovere K, Molenaers G, Van Gestel L, et al. How can push-off be preserved during use of an ankle foot orthosis in children with hemiplegia? A prospective controlled study. Gait Posture. 2006;24(2): 142-151.

5. Gök H, Küçükdeveci A, Altinkaynak H, Yavuzer G, Ergin S. Effects of ankle-foot orthoses on hemiplegic gait. Clin Rehabil. 2003;17(2): 137-139.

6. Franceschini M, Massucci M, Ferrari L, Agosti M, Paroli C. Effects of an ankle-foot orthosis on spatiotemporal parameters and energy cost of hemiplegic gait. Clin Rehabil. 2003;17(4):368-372.

7. Churchill AJ, Halligan PW, Wade DT. Relative contribution of footwear to the efficacy of ankle-foot orthoses. Clin Rehabil. 2003;17(5):553-557.

8. Maeshima S, Okazaki H, Okamoto S, et al. A comparison of kneeankle-foot orthoses with either metal struts or an adjustable posterior strut in hemiplegic stroke patients. J Stroke Cerebrovasc Dis. 2015; 24(6):1312-1316.

9. Maguire C, Sieben JM, Frank M, Romkes J. Hip abductor control in walking following stroke - the immediate effect of canes, taping and TheraTogs on gait. Clin Rehabil. 2010;24(1):37-45.
10. Thijssen DH, Paulus R, van Uden CJ, Kooloos JG, Hopman MT. Decreased energy cost and improved gait pattern using a new orthosis in persons with long-term stroke. Arch Phys Med Rehabil. 2007;88(2): 181-186.

11. Carda S, Invernizzi M, Cognolato G, Piccoli E, Baricich A, Cisari C. Efficacy of a hip flexion assist orthosis in adults with hemiparesis after stroke. Phys Ther. 2012;92(5):734.

12. Verheyden G, Nieuwboer A, Feys H, Thijs V, Vaes K, De Weerdt W. Discriminant ability of the Trunk Impairment Scale: a comparison between stroke patients and healthy individuals. Disabil Rehabil. 2005; 27(17):1023-1028.

13. Verheyden G, Nieuwboer A, Mertin J, Preger R, Kiekens C, De Weerdt W. The trunk impairment scale: a new tool to measure motor impairment of the trunk after stroke. Clin Rehabil. 2004;18(3):326-334.

14. Katsuhira J, Miura N, Yasui T, Mitomi T, Yamamoto S. Efficacy of a newly designed trunk orthosis with joints providing resistive force in adults with post-stroke hemiparesis. Prosthet Orthot Int. 2016;40(1): 129-136.

15. Beckerman H, Becher J, Lankhorst GJ, Verbeek AM. Walking ability of stroke patients: efficacy of tibial nerve blocking and a polypropylene ankle-foot orthosis. Arch Phys Med Rehabil. 1996;77(11): $1144-1151$.

16. Katsuhira J, Matsudaira K, Oka H, et al. Efficacy of a trunk orthosis with joints providing resistive force on low back load during level walking in elderly persons. Clin Interv Aging. 2016;11:1589.

17. Richards CL, Olney SJ. Hemiplegic gait following stroke. Part II: recovery and physical therapy. Gait Posture. 1996;4(2):149-162.

18. Yung-Hui L, Chih-Yong C. Lumbar vertebral angles and back muscle loading with belts. Ind Health. 1999;37(4):390-397.

19. Winter DA. Biomechanics and Motor Control of Human Movement. 4th ed. Hoboken, NJ: John Wiley \& Sons; 2009.

20. Yamamoto S, Fuchi M, Yasui T. Change of rocker function in the gait of stroke patients using an ankle foot orthosis with an oil damper: immediate changes and the short-term effects. Prosthet Orthot Int. 2011;35(4): 350-359.

21. Tyson SF, Kent RM. Effects of an ankle-foot orthosis on balance and walking after stroke: a systematic review and pooled meta-analysis. Arch Phys Med Rehabil. 2013;94(7):1377-1385.

22. Van Criekinge T, Saeys W, Hallemans A, et al. Trunk biomechanics during hemiplegic gait after stroke: a systematic review. Gait Posture. 2017;54:133-143.

23. Nadeau S, Gravel D, Arsenault AB, Bourbonnais D. Plantarflexor weakness as a limiting factor of gait speed in stroke subjects and the compensating role of hip flexors. Clin Biomech (Bristol, Avon). 1999;14(2): $125-135$.

24. Kito N, Shinkoda K, Yamasaki T, et al. Contribution of knee adduction moment impulse to pain and disability in Japanese women with medial knee osteoarthritis. Clin Biomech (Bristol, Avon). 2010;25(9): 914-919.

25. Gage JR, Deluca PA, Renshaw TS. Gait analysis: principles and applications. J Bone Joint Surg Am. 1995;77(10):1607-1623.
Clinical Interventions in Aging

\section{Publish your work in this journal}

Clinical Interventions in Aging is an international, peer-reviewed journal focusing on evidence-based reports on the value or lack thereof of treatments intended to prevent or delay the onset of maladaptive correlates of aging in human beings. This journal is indexed on PubMed Central, MedLine,

\section{Dovepress}

CAS, Scopus and the Elsevier Bibliographic databases. The manuscript management system is completely online and includes a very quick and fair peer-review system, which is all easy to use. Visit http://www.dovepress. com/testimonials.php to read real quotes from published authors. 